\title{
Exploring Irritant Activity of Some of the Phytochemical Components from Wild Sonchus arvensis (L.) ssp arvensis (D.C.) Kirp herb
}

\author{
Ayesha Asif 1 , M. Asif Saeed² \\ 1 University of Central Punjab, Lahore, Pakistan \\ 2155, Mamdot Block, Mustafa Town, Lahore, Pakistan
}

\begin{abstract}
Irritant activeness of sesquiterpene lactone glycosides, isolated from Sonchus arvensis (L.) ssp arvensis (D.C.) Kirp herb were investigated. These compound 1 to compound 7 were identified as sonchuside-E, sonchuside-F, sonchuside-G, sonchuside-H, sonchuside-I sonchuside-A and pirciside C. Such activity was appraised by open mouse ear assay, estimated by $\mathrm{ID}_{50}$. These sesquiterpene lactones exhibited well-defined irritant responses compared with euphorbium, used as reference compound. Compound-1 (sonchuside-E) appeared to be the most potent and persistent irritant with least $\mathrm{ID}_{50}$ whose reaction lasted for more than 48 hours. Compound-2 (sonchuside-F), Compound-3 (sonchuside-G), compound-5 (sonchuside-I) and compound- 6 (sonchuside-A), revealed an irritant reactions of medium intensity while compound-4 (sonchuside-H) and compound-7 (pirciside C) displayed the least irritant and least persistent reaction. The likely mechanisms of their action were discussed.
\end{abstract}

Keywords: Irritant activity, Sesquiterpene lactone glycosides, Sonchus arvensis.

\section{INTRODUCTION}

Sonchus arvensis (L.) ssp arvensis (D.C.) Kirp (Asteraceae or Compositae) also convoked 'milk thistle' or 'moist sow-thistle', is an insalubrious perennial tumid herb with intensified horizontal root system, 1 to 6 feet eminent excavated stems and produce an acrimonious milky latex ${ }^{1-3}$. The plant acquires indiscreet yellow flowers ${ }^{1,2,3}$. It is broadly distributed as weed in the Punjab plains of Paki-

${ }^{*}$ Corresponding author: M. Asif Saeed, e-mail: moasif1605@yahoo.com

Ayesha Asif ORCID Number: 0000-0002-1387-735X

M. Asif Saeed ORCID Number: 0000-0002-6003-7871

(Received 02 November 2019, accepted 05 November 2019) 
stan, very common along roadsides and in the vicinity of cultivated wheat and paddy fields. ${ }^{4,5}$

Among the phytochemical ingredients from different species of genus Sonchus, alkaloids, saponins, cardiac glycosides, tannins, ascorbic acid and polyphenols ${ }^{6-8,21}$, anthraquinones, cyanogenic glycosides, leucoanthocyanins ${ }^{8-10}$, flavonoids and flavonoid glycosides such as coumarins and hydroxy-coumarins ${ }^{8-19,21}$, mono-acyl-galactosyl-glycerol ${ }^{16}$, isocynaroside ${ }^{17,18}$, phyto-sterols, triterpenes from nonsaponi-fiable lipid fraction ${ }^{19}$ have been isolated and characterized from their different parts. Miyase and Fukushima (1987) on the other hand, quarantined nine sesquiterpene glycosides from the elevated parts of Sonchus oleraceus $\mathrm{L}^{20}$. These were named as sonchusides-A, -B, -C and -D along with five others namely glucozaluzanin-C, macrocliniside-A, crepidiaside-A, picrisides-B and $-\mathrm{C}$ whose structures were established on the bases of chemical and spectral data ${ }^{20}$. Tapan (2016), appraised the presence of ascorbic acid, free phenolic acids, catechin, rutin, quercetin, myrecetin, apigenin and kaempferol in four solvent extracts of leaves of wild $S$. arvensis $^{7}$. Heavy metals such as $\mathrm{Pb}, \mathrm{Fe}$, $\mathrm{Cu}, \mathrm{Zn}, \mathrm{Ni}$ and $\mathrm{Ag}$ in Sonchus asper were also assessed ${ }^{22}$.

The whole herb is known as bitter, diuretic, sedative, hypnotic, diaphoretic, antiseptic, expectorant and employed for chronic fever, useful in cooling, cough, bronchitis, asthma and in pulmonary tuberculosis ${ }^{30,32,33}$. Young elevated parts of this herb are boiled, then fried in a pan or cooked and fabricated in tomato sauce are exploited for these purposes ${ }^{24}$. Its leaves are employed by the indigenous inhabits as sedative ${ }^{34,36}$ while its tea is said to have placid impressions on nerves ${ }^{34,35}$. Its root are also utilized for asthma, coughs and in different chest ailments ${ }^{36,37}$. Constraining consequences of $S$. arvensis extract against bladder stones formation, have also been reported ${ }^{38}$. It was further intimated that the aerial parts of $S$. arvensis herb also restrained bioactive antioxidative phenolic compounds with strong radical scavenging capabilities and reducing power ${ }^{8,39}$.

During Dispatching of $S$. arvensis weed from the cultivated wheat and paddy fields by indigenous people, erythema, itching and rashes, were ascertained on abaxial sides of proletarian's hands. The inauspicious impressions of $S$. arvensis on human beings or on animals have not encountered any attention in Pakistan. No endeavors has been flourished to sequestrate and appraise its harmful constituents. In the present communication, we depicted some of its irritant constituents extracted from methanol. The irritant effect of crude methanol was appraised on albino mice, succeeded by fractionation, to sequestrate and characterize its active compounds, whose effectivity were further evaluated by $\mathrm{ID}_{50}$. 


\section{METHODOLOGY}

\section{General Experimental Procedures}

Unless otherwise declared, all the chemicals used were of analytical grades. Melting points were uncorrected. Optical rotation measurements were calculated with Perkin Elmer 241 digital polarimeter. HR-FABMS and FABMS spectra were compiled on JEOL JMS-700 spectrometer in glycerol. UV spectra were quantified on Hitachi 270-30 spectrophotometer in $\mathrm{MeOH}$, while IR spectra were procured by $\mathrm{KBr}$ discs or as thin film on $\mathrm{NaCl}$ discs on a Pye-Unicam SP 8-400 spectrophotometer. ${ }^{1} \mathrm{H}$ NMR spectra were acquired in DMSO- $\mathrm{d}_{6}$ solvent at $270 \mathrm{MHz}$ using tetramethylsilane (TMS) as an internal standard. ${ }^{13} \mathrm{C} \mathrm{NMR}$ spectra were taken at $75 \mathrm{MH}_{\mathrm{z}}$ on Bruker AM-30o NMR spectrometers at $26 \pm$ $2.5^{\circ} \mathrm{C}$ with 0.2-0.5 mM / ml concentrations of the samples, using $10 \mathrm{~mm}$ tubes. Chemical shifts were calculated in the $\delta$ (ppm), using tetramethylsilane (TMS) as an internal reference. Column chromatography was carried out on silica gel $\mathrm{G}_{60}$ (70-230 mesh from E. Merck Germany). Analytical TLC was performed on silica gel $\mathrm{HF}_{254}$ (from E. Merck Germany) with $0.25 \mathrm{~mm}$ thickness. Spots were visualized by exposure to UV light (at 254/365 nm), or with $\mathrm{I}_{2}$ vapors, or with vanillin $/ \mathrm{H}_{2} \mathrm{SO}_{4}$ reagent or with anisaldehyde/ $\mathrm{H}_{2} \mathrm{SO}_{4}$ reagent ${ }^{39,40}$. Preparatory TLC of the pooled column fractions were also executed on $0.75 \mathrm{~mm}$ thick silica gel $\mathrm{HF}_{254}$ TLC glass plates where the samples were applied as narrow bands. The separated bands were scraped off and eluted with a suitable solvent.

\section{Plant materials}

Whole of the Sonchus arvensis (L.) ssp arvensis (D.C.) Kirp herb (about $8.76 \mathrm{~kg}$ ) were accumulated from wet atrophied areas and around the paddy fields from the vicinity of Shakhupura near Lahore (i.e., central plain areas of Punjab) in July/August 2018. These herbs were authenticated by Prof. Dr. Zaheer-ud-Khan, in-charge herbarium, Department of Botany, Government College University, Lahore. A voucher specimen of the sample (No. P-cog.0154) was deposited in the Herbarium of Pharmacognosy Section, Faculty of Pharmacy, University of Central Punjab, Lahore for further reference. Aerial parts of the herbs were detached from roots, air dried in shade and pulverized to a fine powder.

\section{Extraction and isolation}

Air dried aerial parts of Sonchus arvensis (4.90 kg) were extracted three times with $\mathrm{MeOH}$ in a soxhlet apparatus. The extract was concentrated under reduced pressure and residue was suspended in water. This suspension was then extracted with $\mathrm{Et}_{2} \mathrm{O}$. Water layer was passed through an Amberlite XAD-2 column and $\mathrm{MeOH}$ eluate was concentrated under reduced pressure that succumbed 
$80.23 \mathrm{~g}$ of residue. A portion of residue (46.50 g) was rechromatographed on silica gel column $(4 \times 250 \mathrm{~cm})$. The elution of column was carried out with solvent system of $\mathrm{CHCl}_{3}$ and $\mathrm{CHCl}_{3}-\mathrm{MeOH}$, while increasing the amount of latter solvent gradually. The fractions containing similar compounds were pooled together after monitoring by analytical TLC. The pooled fractions were evaporated to dryness under reduced pressure. Preparative thin layer ( $0.75 \mathrm{~mm}$ thick) chromatography of the pooled column fractions were executed on silica gel by applying the samples as uniform bands and using suitable solvent system. The separated bands were scraped off and eluted with methanol.

\section{Compound 1}

Compound 1 was eluted from column with $\mathrm{CHCl}_{3} / \mathrm{MeOH}$ (95:5) and from preparative TLC (with solvent system $\mathrm{CHCl}_{3} / \mathrm{MeOH}$ 90:10). It appeared as amorphous powder and after re-crystallization with hot EtOH, $490 \mathrm{mg}$ (yield = 1.054\%) of acicular crystals were procured. HR-FABMS m/z: 426.18897[ $\left.\mathrm{M}^{+}\right]$; (Found $\mathrm{C}=56.71 ; \mathrm{H}=7.20 . \mathrm{C}_{21} \mathrm{H}_{30} \mathrm{O}_{9} \cdot \mathrm{H}_{2} \mathrm{O}$ requires $\mathrm{C}=56.75 ; \mathrm{H}=7.26 \%$ ). $[\alpha]$ ${ }^{26}-27.8^{\circ}\left(\mathrm{H}_{2} \mathrm{O} ;{ }_{C} \mathrm{O} 0.49\right) \mathrm{CD}\left(\mathrm{H}_{2} \mathrm{O} ;_{C} 0.49\right)$. UV $\lambda_{\max }{ }^{\mathrm{MeOH}} \mathrm{nm}(\log \varepsilon) 252$ (3.70). $\mathrm{IRv} v_{\max }$ (thin film) $\mathrm{cm}^{-1}: 3512$ (hydroxyl groups), 3082, 2930, $1780((\gamma$-lactone ring), 1720, 1640 (double bonds), 1212, 1020. ${ }^{1} \mathrm{HNMR}, \delta(\mathrm{ppm}): 0.94(3 \mathrm{H}, s$, $\left.\mathrm{H}_{3}-14\right), 1.83\left(1 \mathrm{H}, t, J=11 \mathrm{H}_{\mathrm{z}}, \mathrm{H}-5\right), 3.71\left(1 \mathrm{H}, d d, J=11.6 \mathrm{H}_{\mathrm{z}}, \mathrm{H}-1\right), 3.80(1 \mathrm{H}, t$, $\left.J=11 \mathrm{H}_{\mathrm{z}}, \mathrm{H}-6\right), 4.87\left(1 \mathrm{H}, d, J=6 \mathrm{H}_{\mathrm{z}}\right.$, anomeric proton $), 5.28\left(1 \mathrm{H}, d, J=3.2 \mathrm{H}_{\mathrm{z}}\right.$, $\mathrm{H}-13 \mathrm{a}), 6.10\left(1 \mathrm{H}, d, J=3.6 \mathrm{H}_{\mathrm{z}}, \mathrm{H}-13 \mathrm{~b}\right), 9.71\left(1 \mathrm{H}, d, J=4 \mathrm{H}_{\mathrm{z}}, \mathrm{H}-15\right) .{ }^{13} \mathrm{CNMR}$, $\delta(\mathrm{ppm})$ : Aglycone moiety indicated at 83.5(C-1), 25.7(C-2), 24.9(C-3), 48.9(C4), 50.4(C-5), 82.2(C-6), 49.6(C-7), 21.8(C-8), 36.9(C-9), 41.1(C-10), 140.5(C11), 169.9(C-12), 117.5 (C-13), 12.9(C-14), 203.2(C-15); sugar moiety demonstrated 102.3(C-1), 75.3(C-2), 78.4(C-3), 72.4(C-4), 78.8(C-5), 63.7(C-6). The compound 1 exhibited eudesmanolide-type skeleton and was identified by comparing its spectral data with reported data ${ }^{41-43}$ and with CAS ID $=$ Coo013017 as sonchuside E (Fig. 1). 

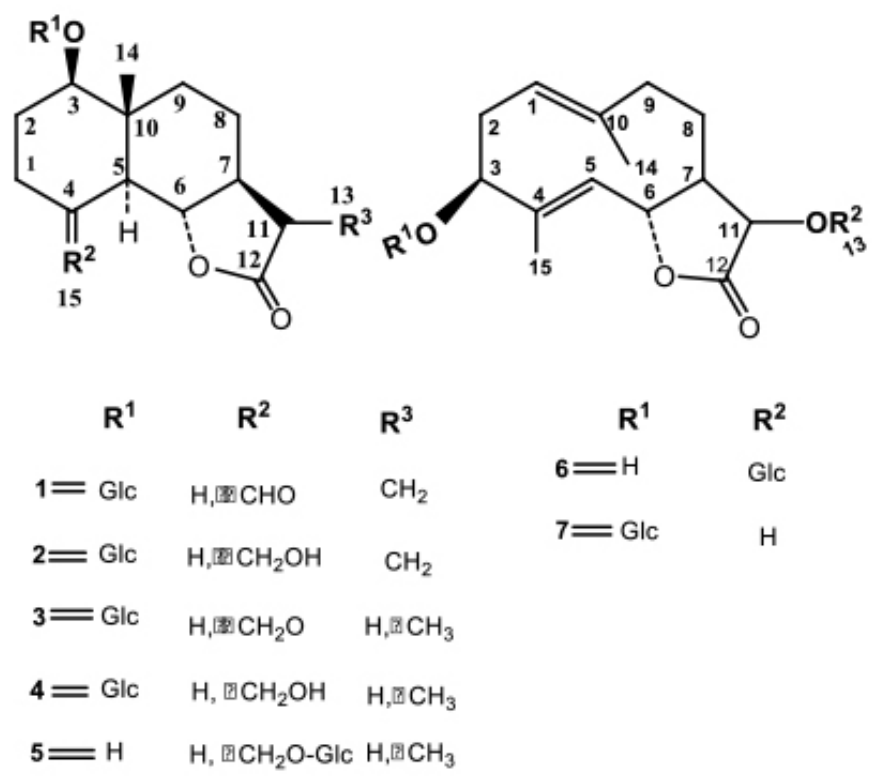

\section{Fig 1. Compounds isolated from Dried Sonchus arvensis herb}

\section{Compound 2}

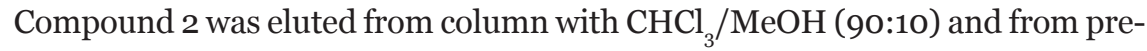
parative TLC (with solvent system $\mathrm{CHCl}_{3} / \mathrm{MeOH} 80: 20$ ). It was visualized as an amorphous light-yellow powder and after re-crystallization with hot $\mathrm{Et}_{2} \mathrm{O} /$ EtOH (50:50 mixture), $134 \mathrm{mg}$ (yield $=0.288 \%$ ) of light yellow colored purified compound was obtained. HR-FABMS m/z: 428.20471 $\left[\mathrm{M}^{+}\right]$. (Found $\mathrm{C}=56.41$, $\mathrm{H}=7.66 . \mathrm{C}_{21} \mathrm{H}_{32} \mathrm{O}_{9} \mathrm{H}_{2} \mathrm{O}$ requires $\left.\mathrm{C}=56.49, \mathrm{H}=7.68 \%\right) \cdot[\alpha]_{\mathrm{D}}{ }^{26}-5 \cdot 9^{\circ}\left(\mathrm{MeOH} ;{ }_{C}\right.$ o.64); $\mathrm{CD}\left(\mathrm{MeOH} ;{ }_{C} 0.34\right)$. UV $\lambda_{\max }{ }^{\mathrm{MeOH}} \mathrm{nm}(\log \varepsilon) 252$ (2.70). IRv ${ }_{\max }$ (thin film) $\mathrm{cm}^{-1}: 3502$ (hydroxyl groups), 2902, 1763 ( $\gamma$-lactone ring), 1717, 1652 (double bonds), 1232, 1024. ${ }^{1} \mathrm{HNMR}, \delta$ (ppm): $0.95\left(3 \mathrm{H}, s, \mathrm{H}_{3}-14\right)$ tertiary methyl signal, $4.84\left(1 \mathrm{H}, d, J=8 \mathrm{H}_{\mathrm{z}}\right.$, anomeric proton), $5.24\left(1 \mathrm{H}, d, J=3.2 \mathrm{H}_{\mathrm{z}}, \mathrm{H}-13 \mathrm{a}\right)$ and $6.16\left(1 \mathrm{H}, d, J=3.6 \mathrm{H}_{\mathrm{z}}, \mathrm{H}-13 \mathrm{~b}\right)$, two olefinic proton signals, which are characteristic of an exocyclic- $\alpha$-methylene- $\gamma$-lactone. ${ }^{13} \mathrm{CNMR}, \delta(\mathrm{ppm})$ : Aglycone moiety indicated by $84.8(\mathrm{C}-1), 28.9(\mathrm{C}-2), 26.7(\mathrm{C}-3), 40.2(\mathrm{C}-4), 50.9(\mathrm{C}-5)$, 84.2(C-6), 50.6(C-7), 21.9(C-8), 37.6(C-9), 42.4 (C-10), 141.8(C-11), 171.6(C12), 115.8(C-13), 13.7(C-14), 66.6(C-15); sugar moiety showed at 102.8(C-1), 75.6(C-2), 78.6(C-3), 72.6(C-4), 78.7(C-5), 63.6(C-6). The compound 2 was identified by comparing its spectral data with reported data ${ }^{41-43}$ and with CAS ID = Coo013013 as sonchuside F (Fig. 1). 


\section{Compound 3}

Compound 3 was eluted from column with $\mathrm{CHCl}_{3} / \mathrm{MeOH}$ (80:20) and from preparative TLC (with solvent system $\mathrm{CHCl}_{3} / \mathrm{MeOH} 70$ :25). It came out as an amorphous colorless powder and after re-crystallization with hot EtOH, 106 $\mathrm{mg}$ (yield $=0.228 \%$ ) of colorless purified compound was obtained. HR-FABMS $\mathrm{m} / \mathrm{z}: 428.20463\left[\mathrm{M}^{+}\right]$. (Found $\mathrm{C}=53.28, \mathrm{H}=7.87 . \mathrm{C}_{21} \mathrm{H}_{32} \mathrm{O}_{9} \cdot \mathrm{H}_{2} \mathrm{O}$ requires $\mathrm{C}$ $=53.29, \mathrm{H}=7.88 \%) .[\alpha]_{\mathrm{D}}{ }^{26}-37.9^{\circ}\left(\mathrm{H}_{2} \mathrm{O} ;{ }_{C} 1.82\right) . \mathrm{UV} \lambda_{\max }{ }^{\text {мeоH }} 252 \mathrm{~nm} . \mathrm{IR} v_{\max }$ (thin film) $\mathrm{cm}^{-1}: 3422$ (hydroxyl groups), 3082, 2932, 1771 ( $\gamma$-lactone ring), 1726, 1652 (double bonds), 1224, 1028. ${ }^{1} \mathrm{HNMR}, \delta(\mathrm{ppm})$ : 0.94 (3H, $\left.s, \mathrm{H}_{3}-14\right)$ tertiary methyl signal, $1.14\left(3 \mathrm{H}, d, J=7 \mathrm{H}_{z}, \mathrm{H}-13\right), 4.81\left(1 \mathrm{H}, d, J=8 \mathrm{H}_{z}\right.$, anomeric proton), $9.68\left(1 \mathrm{H}, d, J=4 \mathrm{H}_{z}, \mathrm{H}-15\right) .{ }^{13} \mathrm{CNMR}, \delta(\mathrm{ppm})$ : Aglycone moiety indicated at $83.4(\mathrm{C}-1), 25.8(\mathrm{C}-2), 24.6(\mathrm{C}-3), 49.2(\mathrm{C}-4), 49.4(\mathrm{C}-5), 81.9(\mathrm{C}-6)$, 53.3(C-7), 23.6(C-8), 37.6(C-9), 42.2(C-10), 41.6(C-11), 177.6(C-12), 12.8(C-13), 12.4(C-14), 24.2(C-15); sugar moiety showed at 102.4(C-1), 75.5(C-2), 78.6(C3), 72.3(C-4), 78.7(C-5), 63.4(C-6). The compound 3 was recognized by comparing its spectral data with reported data ${ }^{41-43}$ and with CAS ID $=$ Cooo13018 as sonchuside G (Fig. 1).

\section{Compound 4}

Compound 4 was eluted from column with $\mathrm{CHCl}_{3} / \mathrm{MeOH}$ (70:25) and from preparative TLC (with solvent system $\mathrm{CHCl}_{3} / \mathrm{MeOH} 70: 20$ ). It came out as an amorphous colorless powder and after re-crystallization with hot $\mathrm{CHCl}_{3} / \mathrm{EtOH}$ (50:50 mixture), $79 \mathrm{mg}$ (yield $=0.172 \%$ ) of colorless purified compound was obtained. HR-FABMS m/z: 430.22030[M+ $]$. (Found $\mathrm{C}=56.09, \mathrm{H}=8.10 . \mathrm{C}_{21} \mathrm{H}_{34} \mathrm{O}_{9}$ $\mathrm{H}_{2} \mathrm{O}$ requires $\left.\mathrm{C}=56.12, \mathrm{H}=8.15 \%\right) .[\alpha]_{\mathrm{D}}{ }^{26}-38.2^{\circ}\left(\mathrm{MeOH} ;{ }_{C} \mathrm{O} .23\right)$. $\mathrm{UV} \lambda_{\max }$ меон $252 \mathrm{~nm}$. IR $v_{\text {max }}$ (thin film) $\mathrm{cm}^{-1} 3414$ (hydroxyl groups), 2924, 1782 ( $\gamma$-lactone ring), 1643 (double bonds), 1158, 1026, 992. ${ }^{1} \mathrm{HNMR} \delta$ (ppm): 0.96 (3H, s, $\mathrm{H}_{3}-$ 14) tertiary methyl signal, $1.09\left(3 \mathrm{H}, d, J=7 \mathrm{H}_{z}, \mathrm{H}-13\right)$ a doublet methyl signal, $4.88\left(1 \mathrm{H}, d, J=8 \mathrm{H}_{z}\right.$, anomeric proton). ${ }^{13} \mathrm{CNMR} \delta(\mathrm{ppm})$ : Aglycone moiety indicated by $84.6(\mathrm{C}-1), 28.8(\mathrm{C}-2), 26.9(\mathrm{C}-3), 39.4(\mathrm{C}-4), 50.3(\mathrm{C}-5), 82.6(\mathrm{C}-6)$, 52.6(C-7), 22.6(C-8), 37.8(C-9), 41.9(C-10), 41.2(C-11), 180.6(C-12), 12.9(C-13), 13.7(C-14), 74.5(C-15); sugar moiety showed by 102.6(C-1), 75.4(C-2), 78.6(C3), 72.3(C-4), 78.4(C-5), 63.3C-6). The compound 4 was agnized by comparing its spectral data with reported data ${ }^{4-43}$ and with CAS ID = Cooo13014 as sonchuside H (Fig. 1).

\section{Compound 5}

Compound 5 was eluted from column with $\mathrm{CHCl}_{3} / \mathrm{MeOH}$ (70:30) and with preparative TLC (with solvent system $\mathrm{CHCl}_{3} / \mathrm{MeOH}$ 60:30). It came out as an 
amorphous yellowish white powder and after re-crystallization with hot $\mathrm{CHCl}_{3} /$ EtOH (40:60 mixture), $38 \mathrm{mg}$ (yield $=0.082 \%$ ) of colorless purified compound was obtained. HR-FABMS m/z: 430.22029[M+H] $]^{+}$. FABMS m/z: (rel. int.): $430[\mathrm{M}+\mathrm{H}]^{+}(76), 415(11), 369(15), 269(100) . \mathrm{MF}=\mathrm{C}_{21} \mathrm{H}_{34} \mathrm{O}_{9} \cdot[\alpha]_{\mathrm{D}}{ }^{26}-31.6^{\circ}$ (MeOH; ${ }_{C}$ O.53). UV $\lambda_{\text {max }}{ }^{\text {MeOH }} 253 \mathrm{~nm}$. IRv $v_{\text {max }}(\mathrm{KBr}) \mathrm{cm}^{-1}: 3512$ (hydroxyl groups), 2884, 1762 ( $\gamma$-lactone ring), 1714, 1642 (double bonds), 1222, 1022. ${ }^{1} \mathrm{HNMR}$ $\delta(\mathrm{ppm}): 1.03\left(3 \mathrm{H}, s, \mathrm{H}_{3}-14\right)$ tertiary methyl signal, $1.16\left(3 \mathrm{H}, d, J=7 \mathrm{H}_{z}, \mathrm{H}-13\right)$ a doublet methyl signal, $3.58\left(1 \mathrm{H}, d d, J=10.6 \mathrm{H}_{z}, \mathrm{H}-1\right), 4.96\left(1 \mathrm{H}, d, J=8 \mathrm{H}_{\mathrm{z}}\right.$ anomeric proton). ${ }^{13} \mathrm{CNMR} \delta(\mathrm{ppm})$ : Aglycone moiety indicated $78.6(\mathrm{C}-1), 31.8(\mathrm{C}-$ 2), 28.9(C-3), 38.0(C-4), 49.9(C-5), 83.2(C-6), 55.5(C-7), 23.7(C-8), 37.9(C-9), 42.8(C-10), 40.9(C-11), 178.6(C-12), 12.8(C-13), 13.2(C-14), 73.8(C-15); sugar moiety showed 103.6(C-1), 75.6 (C-2), 78.4(C-3), 72.2(C-4), 78.4(C-5), 63.3(C6). The compound 5 was accredited by comparing its spectral data with the reported data ${ }^{41-43}$ and with CAS ID = Cooo13015 as sonchuside I (Fig. 1).

\section{Compound 6}

Compound 6 was eluted from column with $\mathrm{CHCl}_{3} / \mathrm{MeOH}$ (70:35) and with preparative TLC (with solvent system $\mathrm{CHCl}_{3} / \mathrm{MeOH}$ 60:40). It came out as a very light yellowish white powder and after re-crystallization with hot $\left(\mathrm{CH}_{3}\right)_{2} \mathrm{O} /$ EtOH (75:25), $32 \mathrm{mg}$ (yield = 0.069\%) of light yellowish white fine acerose purified compound was procured. HR-FABMS m/z: 412.2098[M+H]+. FABMS m/z (rel. int.) $412[\mathrm{M}+\mathrm{H}]^{+}$(22), 397 (21), 387 (15), 329(26), 307(37), 269(64). MF $=\mathrm{C}_{21} \mathrm{H}_{32} \mathrm{O}_{8}$. $[\alpha]_{\mathrm{D}}{ }^{26}-43 \cdot 2^{\circ}\left(\mathrm{MeOH} ;{ }_{C} \mathrm{O} .60\right) . \mathrm{UV} \lambda_{\max } \mathrm{nm}(\log \varepsilon): 279$ (3.75). IR $v_{\max }$ $(\mathrm{KBr}) \mathrm{cm}^{-1}: 3492$ (hydroxyl groups), 2942, 1766 ( $\gamma$-lactone ring), 1722, 1600 (double bonds), 1222, 1026. ${ }^{1} \mathrm{H}-\mathrm{NMR}, \delta$ (ppm): 1.02 and 1.04 (each $3 \mathrm{H}, s_{3} \mathrm{H}_{3}-11$ $\mathrm{H}_{3}$-12) due to methyl group, $2.09\left(3 \mathrm{H}, d, J=6 \mathrm{H}_{z}, \mathrm{H}-13\right), 2.27(3 \mathrm{H}, s, \mathrm{H}-10), 4.12$ $\left(1 \mathrm{H}, d t, J=12.4 \mathrm{H}_{z}, \mathrm{H}-3\right), 4.36\left(1 \mathrm{H}, d, J=4 \mathrm{H}_{z}, \mathrm{H}-4\right), 4.96\left(1 \mathrm{H}, d, J=8 \mathrm{H}_{\mathrm{z}}\right.$, anomeric proton), $6.08\left(1 \mathrm{H}, d, J=18 \mathrm{H}_{\mathrm{z}}, \mathrm{H}-8\right), 7.19\left(1 \mathrm{H}, d, J=16 \mathrm{H}_{\mathrm{z}}, \mathrm{H}-7\right) .{ }^{13} \mathrm{CNMR}$, $\delta(\mathrm{ppm})$ : Aglycone moiety indicated 37.6(C-1), 43.4(C-2), 65.4(C-3), 84.4(C-4), 130.9(C-5), 139.7(C-6), 142.0(C-7), 133.6(C-8), 197.7(C-9), 27.7(C-10), 27.8(C11), 30.2(C-12), 19.9(C-13); sugar moiety showed 106.9(C-1), 75.3(C-2), 78.6(C3), $71.7(\mathrm{C}-4), 78.6(\mathrm{C}-5), 62.8(\mathrm{C}-6)$. The compound 6 was identified by comparing its spectral data with reported data ${ }^{4-47}$ and with CAS ID $=$ Cooo3217O as sonchuside A (Fig. 1).

\section{Compound 7}

Compound 7 was eluted from column with $\mathrm{CHCl}_{3} / \mathrm{MeOH}$ (60:40) and with preparative TLC (with solvent system $\mathrm{CHCl}_{3} / \mathrm{MeOH} 55$ :45). It came out as a fine lustrous powder and after re-crystallization with hot $\mathrm{CHCl}_{3} / \mathrm{EtOH}$ (30:70 mixture), $26 \mathrm{mg}$ (yield $=0.0602 \%$ ) of white fine crystalline mass of purified com- 
pound was obtained. HR-FABMS m/z: 410.19386[M+H]+. FABMS m/z (rel. int.) $410[\mathrm{M}]^{+}$(6o), 354 (17), 340 (21), 270 (18). $\mathrm{MF}=\mathrm{C}_{21} \mathrm{H}_{30} \mathrm{O}_{8} \cdot[\alpha]_{\mathrm{D}}{ }^{26}-63.5^{\circ}$ $\left(\mathrm{MeOH} ;_{C}\right.$ 0.49). UV $\lambda_{\text {max }} \mathrm{nm}(\log \varepsilon): 278$ (3.62). IRv $v_{\text {max }}$ (thin film) $\mathrm{cm}^{-1}: 3454$ (hydroxyl groups), 2922, 1764 (ester group), 1666, 1552, (double bonds)1452, 1408, 1290, 1248, 1146, 1082, 972, 938. ${ }^{1} \mathrm{HNMR}, \delta(\mathrm{ppm}): 1.34$ (3H, br s, H3-14), 1.98 (3H, br s, H3-15), 5.57 (1H, d, J=3.1Hz, H-13a), 6.26(1H, d, J = 16Hz, H-8), $6.41(1 \mathrm{H}, \mathrm{d}, J=3.4 \mathrm{~Hz}, \mathrm{H}-13 \mathrm{~b}), 7.29(1 \mathrm{H}, \mathrm{d}, \mathrm{J}=16 \mathrm{~Hz}, \mathrm{H}-7) .{ }^{13} \mathrm{CNMR}, \delta(\mathrm{ppm})$ : 12.8(C-15), 16.5(C-14), 28.6(C-8), 33.8(C-2), 41.5(C-9), 50.3(C-7), 63.2(C-6'), 71.9(C-4'), 75.5(C-2'), 78.6, 78.8(C-3'/C-5'), 81.4(C-6), 83.5(C-3), 102.7(C1'), 119.6(C-13), 125.5(C-1), 127.3(C-5), 137.9(C-10), 140.7(C-4), 142.3(C-11), 170.4(C-12). The compound 7 was identified by comparing its spectral data with the reported data ${ }^{44,45,48}$ and with CAS ID = Cooo12186 as pirciside C (Fig. 1).

\section{Animals}

Albino mice weighing 10 to $15 \mathrm{~g}$ were procured from the Drug Testing Laboratory, Jail Road Lahore, Pakistan. The animals were housed in plastic cages on wood shavings in an animal house in PCSIR Laboratories, Ferozepure Road Lahore. Six mice were managed per cage in a laminar air flow room maintained under a temperature $28 \pm 2.5^{\circ} \mathrm{C}$ and relative humidity $35 \pm 4.2 \%$. Palette food and de-ionized water were available ad libitum.

\section{Irritant activity}

$10 \mathrm{mg}$ of the test compound was dissolved in $10 \mathrm{ml}$ of acetone to prepare 10 $\mathrm{mg} / 10 \mathrm{ml}(\mathrm{w} / \mathrm{v})$ solution. It was further diluted according to the method of Evans and Schmidt ${ }^{49}$ and Kinghorn and Evans ${ }^{50}$. Ten dilutions were disposed for the main assay. The main procedure for appraising the irritancy on mouse ears was also espoused from Evans and Schmidt ${ }^{49}$ and Kinghorn and Evans ${ }^{50}$. For the main assay, a group of 12 animals were used for each dilution. $5 \mu$ l of one of the dilutions under test was employed to the inner surface of one of the mouse ears using Drummond Microcaps (Drummond Scientific Co. USA), while other ear was regarded as negative control which was without any testing material. Other nine consecutive dilutions were employed substitutable for other groups of animals. 10mg/10ml of euphorbium (a resin from Euphorbia helioscopia) ${ }^{49,50}$ in acetone was employed as a positive control group. Euphorbium was chromatographically purified by column chromatography, prior to its use. Each of the ears was probed for redness after 30 minutes, then after every 15-minute intervals until two observations displayed that further inflammation would not be anticipated. Time of maximum erythema was detected. Number of ears educing the degree of redness agreed with at least ++ intensity on Hecker's scale at peak irritancy ${ }^{51}$ were observed and expressed in $\mu \mathrm{g} / 5 \mu \mathrm{l}$ per ear. Ears of the animals 
were also examined after 24, 48 and 72 hours, to find out any assiduous irritant effectuate of the test compounds. Number of red ears with at least ++ strength (denoted by IU - Irritant units on Hecker scale) ${ }^{51}$ were commemorated after these times. If no redness was detected, the process was reiterated with higher concen-trations of the test solution on ears of another group of animals. Total number of red ears per dilution were tabulated. ID $_{50}$ (Irritant doses in $50 \%$ individuals) were calculated by probit analysis ${ }^{52}$, using a computer program ${ }^{53}$. Number of inflamed mice ears induced by the seven isolated compounds and euphorbium (positive control), their $\mathrm{ID}_{50}, \chi^{2}$, time of ++ irritant reaction, upper and lower confident limits have been outlined in Table 1

Table 1. Mice with positive irritant responses after testing with compounds 1-7

\begin{tabular}{|c|c|c|c|c|c|c|c|c|c|}
\hline \multicolumn{2}{|c|}{$\begin{array}{c}\text { Dose levels } \\
(\mu \mathrm{g} / 5 \mu \mathrm{l})\end{array}$} & $\begin{array}{c}\text { Comp. } \\
1\end{array}$ & $\begin{array}{c}\text { Comp. } \\
2\end{array}$ & $\begin{array}{c}\text { Comp. } \\
3\end{array}$ & $\begin{array}{c}\text { Comp. } \\
4\end{array}$ & $\begin{array}{c}\text { Comp. } \\
5\end{array}$ & $\begin{array}{c}\text { Comp. } \\
6\end{array}$ & $\begin{array}{c}\text { Comp. } \\
7\end{array}$ & $\begin{array}{l}\text { Euphor- } \\
\text { bium }\end{array}$ \\
\hline & - & & - & $9 / 12$ & $10 / 12$ & $11 / 12$ & - & $12 / 12$ \\
\hline \multicolumn{2}{|l|}{5.0} & $12 / 12$ & $11^{*} / 12^{\dagger}$ & 9/12 & $8 / 12$ & $9 / 12$ & $9 / 12$ & $9 / 12$ & $12 / 12$ \\
\hline \multicolumn{2}{|l|}{2.50} & $11 / 12$ & $10 / 12$ & $9 / 12$ & $7 / 12$ & $9 / 12$ & $8 / 12$ & $6 / 12$ & $12 / 12$ \\
\hline \multicolumn{2}{|l|}{1.25} & $9 / 12$ & $9 / 12$ & 8/12 & $6 / 12$ & $8 / 12$ & $6 / 12$ & $4 / 12$ & $12 / 12$ \\
\hline \multicolumn{2}{|l|}{0.625} & $9 / 12$ & $7 / 12$ & 7/12 & $5 / 12$ & $6 / 12$ & $5 / 12$ & $3 / 12$ & $9 / 12$ \\
\hline \multicolumn{2}{|c|}{0.3125} & 8/12 & $5 / 12$ & 7/12 & $3 / 12$ & $5 / 12$ & $4 / 12$ & $2 / 12$ & $8 / 12$ \\
\hline \multicolumn{2}{|c|}{0.15625} & $7 / 12$ & $3 / 12$ & $6 / 12$ & $2 / 12$ & $4 / 12$ & $4 / 12$ & $1 / 12$ & $7 / 12$ \\
\hline \multirow{2}{*}{\multicolumn{2}{|c|}{$\begin{array}{l}0.078125 \\
0.0390625\end{array}$}} & $6 / 12$ & $2 / 12$ & $5 / 12$ & $1 / 12$ & $3 / 12$ & $2 / 12$ & $0 / 12$ & $6 / 12$ \\
\hline & & $3 / 12$ & $1 / 12$ & $3 / 12$ & $0 / 12$ & $2 / 12$ & $1 / 12$ & - & $3 / 12$ \\
\hline \multirow{2}{*}{\multicolumn{2}{|c|}{$\begin{array}{l}0.01953121 \\
0.00976562\end{array}$}} & $2 / 12$ & $1 / 12$ & $0 / 12$ & $\longrightarrow$ & $2 / 12$ & $0 / 12$ & - & $2 / 12$ \\
\hline & & $1 / 12$ & $0 / 12$ & & 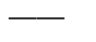 & $1 / 12$ & & 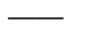 & $2 / 12$ \\
\hline \multirow{6}{*}{$I D_{50}$} & $\mu \mathrm{g} / 5 \mu \mathrm{l}$ & 0.135 & 0.857 & 0.318 & 1.571 & 0.515 & 0.782 & 2.054 & 0.099 \\
\hline & S. D. & 0.099 & 0.099 & 0.187 & 0.139 & 0.149 & 0.106 & 0.204 & 0.094 \\
\hline & $x^{2}$ & 2.102 & 0.738 & 3.732 & 0.931 & 0.531 & 3.286 & 0.713 & 2.943 \\
\hline & $t$ & $1.0 \mathrm{~h}$ & $2.5 \mathrm{~h}$ & $1.5 \mathrm{~h}$ & $4.0 \mathrm{~h}$ & $1.5 \mathrm{~h}$ & $2.5 \mathrm{~h}$ & $4.5 \mathrm{~h}$ & $30 \mathrm{~min}$ \\
\hline & U.C.L. & 0.235 & 1.498 & 0.72 & 3.475 & 1.142 & 1.453 & 5.047 & 0.165 \\
\hline & L.C.L. & 0.073 & 0.513 & 0.141 & 0.858 & 0.241 & 0.442 & 1.195 & 0.056 \\
\hline \multirow{2}{*}{$\begin{array}{c}\text { IU } \\
\text { after }\end{array}$} & $24 \mathrm{~h}$ & 1.26 & 2.5 & 1.25 & 5 & 2.5 & 2.5 & 5 & 1.25 \\
\hline & $48 \mathrm{~h}$ & 2.5 & 5 & 2.5 & $>10$ & 5 & 5 & $>10$ & 2.5 \\
\hline
\end{tabular}

Where:

${ }^{*}$ Number of animal ears reacted to irritant compound. ${ }^{\dagger}$ Total number of animals used.

Comp. 1 = sonchuside E; Comp. 2 = sonchuside F; Comp. $\mathbf{3}$ = sonchuside G;

Comp. 4 = sonchuside H; Comp. 5 = sonchuside I; Comp. 6 = sonchuside A; 
Comp. 7 = pirciside C.. ID $_{50}=$ Irritant dose in $50 \%$ individuals;

S.D. = Standard deviation; $\chi^{2}=$ Chi square $; t=$ Time of maximum irritant reaction;

IU = Irritant units; U.C.L. = Upper confident limits; L.C.L. = Lower confident

Limits. $\mathrm{h}=$ hours after application.

\section{RESULTS AND DISCUSSION}

Methanol extract of the dried $S$. arvensis herb succumbed crude fraction that mortified sesquiterpene lactone glycosides. First endeavour of direct chromatographic analysis of this mixture into pure components was not successful, it was further fractionated into water and ether soluble fractions. Aqueous fraction after passing through an Amberlite XAD-2 column was eluded with $\mathrm{MeOH}$ and re-chro-matographed on silica gel column and preparative thin layers, which afforded seven sesquiterpene lactone glycosides (compound 1 to 7 ). All these compounds were identified by comparison of their chromatographic and spectroscopic data with the published values ${ }^{41-48}$. It is known that sesquiterpene lactones present in various species of family Asteraceae are pledged in a number of functions. A panoptic spectrum of their biological activeness have been reported ${ }^{19,20,26,27,32-34,37,38}$. The structure-activity relationships (SAR) of these lactones ascertained that the mien of an $\alpha$-methylene exocyclic to a $\gamma$-lactone ring is substantive for most of their stringy toxic pharmacological and biological activeness, especially when such compounds are prosecuted on the human and animal's skins ${ }^{49,50,54-56}$. This is staunch for such active group, when an inflammatory reaction due to irritancy, allergenicity or cross-sensitivity are pertained. It has also been investigated that $\mathrm{C}=\mathrm{C}$ grouping conjugated to a lactone, is an immunological prerequisite ${ }^{49,50,54-56}$. Plant extracts are frequently a complex mixture of phytochemical compounds that prosecute on skin by dissimilar mechanisms, with different effectivity and continuance of action ${ }^{55,56}$. Mouse ear test is known to be invaluable for screening various plant extracts for inflammatory reaction ${ }^{49-51}$. For comparability of the irritant activities of $S$. arvensis's sesquiterpene lactones, the total number of mice, indicating inflammatory reaction were enumerated at the time of peak irritancy. The data was analyzed by computer program ${ }^{52,53}$ which enable us to draw a comparison between the potentialities by means of $\mathrm{ID}_{50}$ that afford plenteous assurance, because limits were located on upper and lower confident levels, along with the standard deviation. Standard deviation also manoeuver to quantify the slop of probit regression line which intimated the overall 'shape' of Gaussian distribution of tolerance curve ${ }^{52}$. The intention of $\chi^{2}$ test, computed by the probit program, 
was to ascertain whether the results of the assay after transformation, were appropriately symbolized by the probit regression line ${ }^{52}$. All the abstracted seven sesquiterpene lactone glycosides paraded irritant effectuates on the mice's ears after comparison with euphorbium reaction. In all the cases, the reaction first came out as red inflamed area (erythema). The profound response then turned to a scale formation, followed by oedema. Redness of the mouse's ear appeared between twenty minutes to about two hours after assiduity and in some cases, it reached to a maximum level of ++++ intensity in about four hours. Later on, oedema of the ears was espoused by a little transudation of watery fluid from the notorious skin. Compound 1(sonchuside-E) revealed more escalated irritant reaction than all the other six compounds, with minimum $\operatorname{ID}_{50}($ Table 1.). Compounds 2 (sonchuside-F), -3(sonchuside-G), -5 (sonchuside-I) and -6 (sonchuside-A) imparted an irritant reactions of medium vividness, while compounds -4 (sonchuside- $\mathrm{H}$ ) and -7 (pirciside $\mathrm{C}$ ) revealed the least irritant chroma on the mice ears, when compared with the euphorbium reaction (Table 1.). The results further betoken that the irritant reactions of these sesquiterpene lactones litigated up to 24 or 48 hours. The inauspicious reaction of compounds-1 (sonchuside-E) was continued even languished more than 48 hours, in a similar way as the reaction revealed by euphorbium (Table 1.). The presence of $\alpha$-methylene group exocyclic to $\gamma$-lactone ring in these sesquiterpene lactone glycosides from $S$. arvensis was plausibly the main causation of scaly dermatitis of irritant type ${ }^{49-51}$, within a short time that was lasted up to 48 hours or even more as in case of compound-1 (Table 1., Fig. 1). The pioneering of erythema, scales and oedema was credibly due to the presence of exocyclic oxygen and double bonds present in their molecules (Fig.1) which perhaps constituted some kind of adduct with the skin proteins and as a consequence inflammation and scaling of mouse skin was anticipated. Since compound-1 possessed an exocyclic $\alpha$-methylene- $\gamma$-lactone signals at $\delta 5.28$ and 6.10, an aldehyde proton signal at $\delta 9.71$ and an anomeric proton signal at $\delta 4.87$, the stronger irritation of this compound was probably due to such moieties (Fig. 1). Medium irritant response of compound- 3 compared to others due to the absence of exocyclic methylene proton signals at $\mathrm{C}-13$ and the appearance of a doublet methyl signal at $\delta$ 1.14. The situation in case of compound-7, that have least irritation, might be due to some double bonds present in its molecule (Fig. 1).

Although the definite mechanism of this activity has not been elucidated, these phenomena might be considered to occur, due to the differences in binding affinities of these sesquiterpene lactones on the active sites of proteins or receptors present in animal's skin. We had exploited only that much quantities of these compounds which at least gave ++ reaction on Hecker's scale ${ }^{51}$. The doses 
of the compounds utilized in this work, only caused erythema, scaling and oedema, while in case of compound-1, exudation of water from the implemented area was also perceived, possibly due to some biological damage of skin cells. In all these reactions, hyper-pigmentation was not ascertained. This was likely that due to less quantities of these lactones were able to be penetrated in animal skin and thus were not spendable for hyper-pigmentation.

We concluded that $S$. arvensis herb constrained irritant sesquiterpene lactone glycosides, which could be injurious not only to the animals, but also to the human beings. The despicable and reiterated doses of these compounds, with controlled clinical conditions may be pursued as an anti-inflammatory and sedative agent, as it was arrogated in the folk medicines ${ }^{5,6,20-25}$. Oral presidency of these sesquiterpene lactone glycosides in low and ingeminated doses may be a safer route than topical application for anti-inflammatory and sedative purposes, particularly in case where erythema is of diffused type and whole of the body is involved. On the other hands, the topical application of higher dosages of these compounds might commove the normal pattern of skin cells. These perturbations often induce neoplasmic growth in skin cells, including skin carcinomas. These possibilities necessitate furthermore investigation. Moreover, further substantial work is indispensable to inflate these properties, through the grooming of their derivatives that would possible lead to the structure-activity relationship of such important molecules of natural origin.

\section{ACKNOWLEDGEMENTS}

The author and co-author are thankful to the staff of Pharmacognosy Section, College of Pharmacy, University of the Punjab Lahore, Pakistan and Department of Botany, Government College University Lahore for their help. Special thanks to Prof. Dr. Zaheer-ud-Khan, in-charge herbarium, Department of Botany, GCU. Lahore, Pakistan for authenticating the materials. Another thanks are presented to the staff of Applied Chemistry Section, PCSIR Laboratories, Lahore, Pakistan for procuring HR-FABMS, FABMS, ${ }^{1} \mathrm{HNMR}$ and ${ }^{13} \mathrm{CNMR}$ spectra. 


\section{REFERENCES}

1. Ballero, M.; Poli, F.; Sacchetti, G.; Loi, M. C. Ethnobotanical research in the territory of Fluminimaggiore (south western Sardinia). Fitoterapia 2001, 72, 788-801.

2. Passalacqua, N. G.; Guarrera, P. M.; De, F. G. Contribution to the knowledge of the folk plant medicine in Calabria region (Southern Italy). Fitoterapia 2007, 78, 52-68.

3. Kashyap, S. R.; Joshi, A. C. Compositae, In; Lahore district flora.; University of the Punjab, Lahore, Pakistan; 1936, pp. 124-156.

4. Bakhsh, A.; Hussain, M.; Dasti, A. A.; Mahmood, S.; Din, M. A.; Naqvi, S. M. S. Study of weeds in wheat fields. Pak J. Ari. Agri. 2006, 9, 41-45.

5. Frank, C. R.; Shirley N. H. Herbal remedies of the maritime Indians. A preliminary screening Part III. J. Ethnopharmacol. 1982, 6, 275-285.

6. Tapan, S. Quantitative HPLC analysis of phenolic acids, flavonoids and ascorbic acid in four different solvent extracts of two wild edible leaves, Sonchus arvensis and Oenanthe linearis of North-Eastern region in India. J. Appl. Pharmace. Sci. 2016, 6, 157-166.

7. Rahmat, A. K. Evaluation of flavonoids and diverse antioxidant activities of Sonchus arvensis. Chem. Cent. J. 2012, 6, 126.

8. Bondarenko, V. G.; Glyzin, V. I.; Shelyuto, V. L.; Smirnova, L. P. Flavonoids of Sonchus arvensis L. Khim. Prir. Soedin. 1976b, 7, 542.

9. Bondarenko, V. G.; Glyzin, V. I.; Shelyuto, V. L.; Smirnova, L. P., Flavonoids of Sonchus arvensis. Chem. Nat. Comp. 1977a, 12, 484.

10. Hussain, J.; Muhammad, Z.; Ullah, R.; Khan, F. U.; Khan, I. U.; Khan, N.; Ali, J.; Jan, S. Evaluation of the chemical composition of Sonchus eruca and Sonchus asper. J. Am. Sci. 2010, 6, 231-235.

11. Bondarenko, V. G.; Shelyuto, V. L.; Glyzin, V. I.; Khoronko, A. T. Flavonoids of Sonchus arvensis L. Fitokhim Izuch. Flory BSSR Biofarm. Issled. Lek. Prep. 1977b, 91-92.

12. Bondarenko, V. G.; Glyzin, V. I; Shelyuto, V. L. Sonchoside as a new flavonoid glycoside from Sonchus arvensis L. Khim. Prir. Soedin. 1978, 9, 403.

13. Qu, G.; Liu, J.; Li, X.; Wang, S.; Wu, L.; Li, X. Flavonoids of lieyejumaicai (Sonchus arvensis). Zhongeaoyao, 1995, 26, 233-235.

14. Qu, G.; Li, X; Liu, J. Studies on flavonoid glycosides of Sonchus arvensis L. Zhong guo Zhongyao Zazhi. 1996, 21, 292-294.

15. Putul, B. N. C.; Baruah, R. P.; Sharma, J. N.; Baruah, P. K.; Werner H. A monoacylgalactosyl-glycerol from Sonchus arvensis. Phytochem. 1983, 22, 1741-1744.

16. Bondarenko, V. G.; Glyzin, V. I.; Bankovskii, A. I.; Shelyuta, V. L. Isocinaroside, a new flavone glycoside from Sonchus arvensis L. Khim. Prir. Soedin. 1974, 5, 665.

17. Bondarenko, V. G.; Glyzin, V. I.; Ban'kovskii, A. I.; Shelyuto, V. L. Isocynaroside. A new flavone glycoside from Sonchus arvensis. Chem. Nat. Comp.,1976a, 10, 680-681.

18. Shirley, N. H.; Chandler, R. F. Herbal remedies of the maritime Indians. Phytosterols and triterpenes of 67 plants. J. Ethnopharmacol. 1984, 10, 181-194.

19. Miyase, T.; Fukushima, S. Studies on sesquiterpene glycosides from Sonchus oleraceus L. Chem. Pharm. Bull. 1987, 35, 2869-2874.

20. Rahmat, A.K.; Muhammad, R.K.; Sumaira, S.; Mushtaq, A. Evaluation of phenolic contents and antioxidant activity of various solvent extracts of Sonchus asper (L.) Hill. Chem. 
Cent. J. 2012, 6, 12.

21. Hussain, I.; Ullah, R.; Khan, N.; Ayaz, S.; Ahmad, S.; Shanzeb; Ahmed, M.; Hasan, P. T.; Khan, F. A. Phytochemicals and inorganic profile of Calendula officinale and Sonchus asper. Afr. J. Pharm. Pharmacol. 2011, 5, 1813-1818.

22. Seaman, F. C.; Funk, V. A. Cladistic analysis of complex natural product: Developing transformation series from sesquiterpene lactone data. Taxon. 1983, 31, 1-27.

23. Silverstein, R. M.; Bassler, G. C.; Morrill, T. C. Spectrometric identification of organic compounds. $4^{\text {th }}$ edition, John Wiley and sons, New York, USA. 1981.

24. Williams, D. H.; Fleming, I. Spectroscopic methods in organic chemistry $3^{\text {rd }}$ edition, Mc graw-Hill Book company Ltd., London, UK. 1980.

25. Budzikiewicz, H.; Wilson, J. M.; Djerassi, C. Mass spectrometry in structural and stereochemical problems XXXII. J. Amer. Chem. Soc. 1963, 85, 3688-3699.

26. Fraga, B. M. Natural sesquiterpenoids. A Review. Nat. Prod. Rep. 2007, 24, 1350-1381.

27. Tabatabaei, S. M.; Ebrahimi, S. N.; Salehi, P.; Sonboli, A.; Tabefam, M.; Kaiser, M.; Hamburger, M.; Farimani, M. M. Antiprotozoal germacranolide sesquiterpene lactones from Tanacetum sonbolii. Planta Med. 2019, 85, 424-430.

28. Tabatabaei, M. S.; Salehi, P.; Farimani, M. M.; Neuburger, M.; De-Mieri, M.; Hamburger, M.; Nejad-Ebrahimi, S. A nor-diterpene from Salvia sahendica leaves. Nat. Prod. Res. 2017, 31, 1758-1765.

29. Muhammad, I. S.; Mir, A. K. Folk use of medicinal herbs of Margalla Hills National Park, Islamabad. J. Ethnopharmacol. 2000, 69, 45-56.

30. Alessandro-di, T.; Łuczaj, Ł.; Cassandra, L.; Quave, S. R.; Andrea, P. Traditional food and herbal uses of wild plants in the ancient South-Slavic diaspora of Mundimitar/Montemitro (Southern Italy). J. Ethnobio. Ethnomed. 2012, 8, 21.

31. Jabeen, A.; Khan, M. A.; Ahmad, M.; Zafar, M.; Ahmad, F. Indigenous uses of economically important flora of Margallah Hills National Park, Islamabad, Pakistan. Afri J. Biotech. 20o9, $8,763-784$.

32. Allah, B. G.; Altaf, A. D.; Sabir, H.; Muhammad, I. A. Muhammad, A. D. Indigenous uses of medicinal plants in rural areas of Dera Ghazi Khan, Punjab, Pakistan. ARPN J. Agricul. Bio. Sci. 2012, 7, 750-762.

33. Rahman, A. H. M. M.; Gulsan, J. E.; Alam, M. S.; Ahmad, S.; Naderuzzaman, A. T. M.; Islam A. K. M. R. An ethnobotanical portrait of a village: Koikuri, Dinajpur with reference to medicinal plants. Int. J. Biosci. 2012, 2, 1-10.

34. Mahbubur, A. H. M. R.; Saika, K. N.; Zannatul, F.; Rafiul-Islam, A. K. M. Medico-botany on herbaceous plants of Rajshahi, Banglabesh. Amer. J. Life Sci. 2013, 1, 136-144.

35. Foster, S.; Duke, J. A. A. Field Guide to Medicinal Plants. Eastern and Central N. America; Houghton Mifflin Co.1990, ISBN 0395467225.

36. Singh, A. K.; Raghubanshi, A. S.; Singh, J. S. Medical ethnobotany of the tribals of Sonaghati of Sonbhadra district, Uttar Pradesh, India. J. Ethnopharmacolo. 2oo2, 81, 31-41.

37. Wahjoedi, B.; Nurendah, P. The inhibitative effects of Sonchus arvensis infusion on bladder stone formation in mice. MEDIKA (Medika Kedokteran dan farmasi) 1986, 12, 951-953.

38. Usman, R.; Khan, A.; Gul, S.; Rauf, A.; Muhammad, N. Evaluation of In vitro antioxidant properties of selected medicinal plants. Middle East J. Med. Pl. Res. 2012, 1, 28-31. 
39. Wagner, H.; Bladt, S.; Zgainski, E. M. Plant Drug Analysis. Springer, Berlin, 1984, pp. 299-304.

40. Stahl, E. Thin layer chromatography. Springer-verlag, 1969, Berlin, New York, USA.

41. Mahmoud, Z.; Masry, S. E.; Amer, M.; Ziesche, J.; Bohlmann, F. Two eudesmanolides from Sonchus macrocarpus. Phytochemistry, 1983, 22, 1290-1291.

42. Mahmoud, Z.; Masry, S. E.; Amer, M.; Ziesche, J.; Grenz, M. Sesquiterpene lactones from Sonchus macrocarpus. Phytochemistry, 1984, 23, 1105-1107.

43. Shimizu, S.; Miyase, T.; Ueno, A.; Usmanghani, K. Sesquiterpene lactone glycosides and ionone derivative glycosides from Sonchus asper. Phytochemistry, 1989, 28, 3399-3402.

44. Zidorn, C. Sesquiterpene lactones and their precursors as chemosystematic markers in the tribe Cichorieae of the Asteraceae. A review. Phytochemistry, 2008, 69, 2270-2296.

45. Bitama, F.; Ciavatta, M. L.; Manzo, E.; Dibi, A.; Gavagnin, M. Chemical characterisation of the terpenoid constituents of the Algerian plant Launaea arborescens. Phytochemistry, 2008, 69, 2984-2992.

46. Kisiel, W.; Zielińska, K. Guaianolides from Cichorium intybus and structure revision of cichorium sesquiterpene lactones. Phytochemistry, 2001, 57, 523-527.

47. Warashina, T.; Miyase, T. Sesquiterpenes from the roots of Cichorium endivia. Chem. Pharm. Bull. 2008, 56, 1445-1451.

48. Nishimura, K.; Miyase, T.; Ueno, A.; Noro, T.; Kuroyanagi, M.; Fukushima, S. Sesquiterpene lactones from Picris hieracioides L. Var. Japonica Regel. I. Chem. Pharm. Bull. 1986, $34,2518-2521$.

49. Evan, F. J.; Schmidt, R. J. An assay procedure for the comparative irritancy testing of the esters in the tigliane and daphnane series. Inflammation, 1979, 3, 215-223.

50. Kinghorn, A. D.; Evans, F. J. A biological screen of selected species of the genus Euphorbia for skin irritant effects. Planta Medica, 1975, 28, 325-335.

51. Hecker, E. Isolation and characterization of the co-carcinogenic principles from croton oil. In; Methods of cancer research, Busch, H.; Ed.; Academic press: London, 1971, pp. 439-484.

52. Finney, D. J. Probit Analysis, 3rd edition, Cambridge University Press, London, 1971.

53. Probit Analysis; Biostat for windows ver. 5.9.8.5; AnalystSoft.

54. Schlewer, G.; Stampf, J. L.; Benezra, C. Synthesis of $\alpha$-methylene- $\gamma$-butyrolactones. A structure-activity relationship. Studies of their allergenic power. J. Med. Chem. 1980, 23, 1031-1038.

55. Epstein, W. L.; Reynolds, G. W.; Rodriguez, E. Sesquiterpene lactone dermatitis. Crosssensitivity in costus-sensitized patients. Arch. Dermatol. 1980, 116, 59-60.

56. Mitchell, J. C.; Fritig, B.; Singh, B.; Towers, G. H. N. Allergic contact dermatitis from Frullania and Compositae. The role of sesquiterpene lactones. J. Investig. Dermatol. 1970, 54, 233-239. 